\title{
TRIM14 promotes colorectal cancer cell migration and invasion through the SPHK1/ STAT3 pathway
}

Zhonghai Jin ${ }^{\dagger}$, Hongguang Li ${ }^{\dagger}$, Xiaofei Hong, Guangrong Ying, Xiaofeng Lu, Lilei Zhuang and Shenbao Wu ${ }^{*}$

\begin{abstract}
Background: Colorectal cancer (CRC) is one of the most lethal malignancies. Tripartite Motif Containing 14 (TRIM14) is a member of TRIM family proteins, which are involved in the pathogenesis of various cancers. This study aimed to investigate TRIM14 expression in CRC tissues, and its effects on the migration and invasion of CRC cell lines.

Methods: TRIM14 mRNA expression was detected by real-time PCR analysis. Cell migration and invasion were measured by Transwell assays. Protein expression was assessed by western blot analysis.

Results: The expression of TRIM14 was significantly higher in CRC tissues than in matched non-cancerous tissues. TRIM14 knockdown by specific short hairpin RNA (shRNA) attenuated CRC cell migration and invasion, whereas TRIM14 overexpression caused reverse effect. Moreover, TRIM14 positively regulated the protein levels of sphingosine kinase 1 (SPHK1) and phosphorylated STAT3 (p-STAT3), as well as the mRNA and protein expression of matrix metalloproteinase 2, MMP9 and vascular endothelial growth factor, which are transcriptional targets of the STAT3 signaling pathway. Importantly, the blockage of the SPHK1/STAT3 signaling pathway by SKI-II or AG490 could reverse the TRIM14-promoted CRC cell migration and invasion.
\end{abstract}

Conclusions: Our results reveal a critical role for TRIM14 in promoting migration and invasion of CRC cells, and suggest TRIM14 may serve as a potential molecular target to prevent CRC metastasis.

Keywords: TRIM14, STAT3, SPHK1, Migration, Invasion

\section{Background}

Colorectal cancer (CRC) is one of the most common causes of cancer-related death in many countries. The main reason of high mortality rate in CRC patients is the extreme difficulty in treating distant metastases [1]. The feature characteristics of aggressive metastatic cancers include high ability of migration, consequent invasion and adhesion in the distant organs [2]. Until now, the complex mechanisms that lead to cancer metastasis are far from being fully understood.

Members of the tripartite motif (TRIM) family, also called the RING B-box-coiled-coil (RBCC) family, are involved in the pathogenesis of various cancers by acting as

\footnotetext{
*Correspondence: wushenbao100@163.com

${ }^{\dagger}$ Zhonghai Jin and Hongguang Li contributed equally to this work Department of Gastroenterology, Yiwu Hospital, Wenzhou Medical University, 699 Jiangdong Middle Road, Yiwu 322000, China
}

oncogenes or tumor suppressor genes [3]. A recent study has reported that TRIM14 expression was down-regulated in non-small cell lung cancer (NSCLC) and played a tumor suppressive role in NSCLC progression [4]. Conversely, studies showed that TRIM14 expression was up-regulated in hepatocellular carcinoma (HCC) [5], osteosarcoma [6], oral squamous cell carcinoma (OSCC) [7], tongue squamous cell carcinoma (TSCC) [8], breast cancer [9] and glioma [10], and that TRIM14 overexpression promoted cell proliferation, migration, invasion and chemoresistance, supporting the oncogenic role of TRIM14 in these cancers. However, few reports have focused on the expression and functions of TRIM14 in CRC.

STAT3 belongs to the signal transducer and activator of transcription (STAT) family, which is activated by the upstream stimuli, such as cytokines, growth factors and non-receptor tyrosine kinases. In response to these 
stimuli, STAT3 is phosphorylated at Tyr 705, which induces the dimerization of STAT3 through phosphotyrosine-SH2 domain interaction [11, 12]. The dimerized STAT3 translocates to the nucleus, interacts with specific DNA elements and then stimulates the transcription of target genes [11, 12]. STAT3 is found constitutively activated in many human cancers, which is related to malignant characteristics, including rapid proliferation, migration, invasion and metastasis [11, 13]. The critical role of the STAT3 signaling pathway in CRC progression also has been reported [14]. Sphingosine kinase 1 (SPHK1) catalyzes the formation of sphingosine 1-phosphate (S1P), which promotes tumor growth, angiogenesis and metastasis [15]. SPHK1 is found to be upregulated in $\mathrm{CRC}$, and associated with CRC progression and prognosis [16]. A recent study has reported that SPHK1 leads to the constitutive activation of STAT3 in colitis-associated CRC [17]. TRIM8, another member of TRIM proteins, was found to enhance the STAT3 signaling pathway in various cell types [18-20]. TRIM14 overexpression increased the phosphorylation of STAT3 in breast cancer cells [9], while it was unclear whether TRIM14 was related to the STAT3 signaling during CRC progression.

In the present study, TRIM14 expression was elevated in CRC tissues. Knockdown of TRIM14 repressed CRC cell migration and invasion as well as the levels of phospho-STAT3 (p-STAT3), matrix metalloproteinase 2 (MMP2), MMP9 and vascular endothelial growth factor (VEGF). Importantly, inhibition of STAT3 signaling attenuated TRIM14-enhanced cell invasion and migration of CRC cells. Our findings indicate that TRIM14 represents a novel therapeutic target in CRC.

\section{Materials and methods Patient samples}

The study was approved by the Ethics Committee of Yiwu Hospital, Wenzhou Medical University (Yiwu, China). A total of 40 CRC patients (24 men and 16 women, age range: 33-71 year) who underwent surgical resection at Department of Gastroenterology, Yiwu Hospital were enrolled in this study after written informed consent were obtained from all the patients. During surgery, CRC and matched non-cancerous colorectal tissue samples were obtained. Part of the specimens were subjected to hematoxylin and eosin (HE) staining for histopathological analysis, and the other part of the specimens were immediately snap-frozen and stored at $-80{ }^{\circ} \mathrm{C}$. The histopathological stage of each CRC was determined by the pathologists according to the tumor-node metastasis (TNM) classification system. Clinical characteristics, including gender, age, tumor size and tumor stage, were retrieved from the medical records (Table 1).
Table 1 Clinicopathological characteristics $(n=40)$

\begin{tabular}{lll}
\hline Characteristic & Cases & $\%$ \\
\hline Gender & & \\
Male & 24 & 60.0 \\
Female & 16 & 40.0 \\
Age (years) & & \\
$\geq 65$ & 26 & 65.0 \\
$<65$ & 14 & 35.0 \\
Tumor size (cm) & & \\
$\geq 5.0$ & 21 & 52.5 \\
$<5.0$ & 19 & 47.5 \\
Clinical stage & & \\
I/II & 18 & 45.0 \\
III & 22 & 55.0 \\
\hline
\end{tabular}

\section{Real-time PCR analysis}

The Trizol reagent (Invitrogen Carlsbad, CA, USA) was used to isolate total RNA from the tissue samples and cell lines per the manufacturer's protocol. Complementary DNA (cDNA) was then synthesized from the isolated total RNA with cDNA synthesis kit (Thermo Fisher, Rockford, IL, USA), and used as template in Real-time PCR analysis on an ABI 7300 system (Applied Biosystem, Foster City, CA, USA) with SYBR Green qPCR Master Mixes (Thermo Fisher). The oligonucleotides used as PCR primers are listed in Table 2. Gene expression was normalized to the expression of GAPDH by $2^{-\Delta \Delta C T}$ methods [21].

\section{Cell lines and culture conditions}

Human CRC cell lines SW620, Caco2, LoVo, HT-29 and SW1116 obtained from Shanghai Institute of Biochemistry and Cell Biology (SIBCB, Shanghai, China) were grown at $37{ }^{\circ} \mathrm{C}$ in a humidified atmosphere containing $5 \% \mathrm{CO}_{2}$ and $95 \%$ air. All cell lines were cultured in RPMI1640 (Hyclone, Rockford, IL, USA) plus 10\% fetal bovine serum (FBS, Hyclone) and 100 units/ml penicillin and streptomycin.

Table 2 Primer pairs used for real-time PCR

\begin{tabular}{|c|c|}
\hline Gene & Primers (forward/reverse) \\
\hline TRIM14 & $\begin{array}{l}\text { 5'-GGATTTGTGTCTCCGTTCTG-3' and 5'-TCTGTCTGCCTGGTA } \\
\text { TTCTG-3' }\end{array}$ \\
\hline VEGF & $\begin{array}{l}\text { 5'-ATTTCTGGGATTCCTGTAG-3' and 5'-CAGTGAAGACACCAA } \\
\text { TAAC-3' }\end{array}$ \\
\hline MMP2 & $\begin{array}{l}5^{\prime} \text {-TACAGGATCATTGGCTACACACC-3' and } 5^{\prime} \text {-GGTCACATCGCT } \\
\text { CCAGACT-3' }\end{array}$ \\
\hline MMP9 & $\begin{array}{l}\text { 5'-TGTACCGCTATGGTTACACTCG-3' and 5'-GGCAGGGACAGT } \\
\text { TGCTTCT-3' }\end{array}$ \\
\hline GAPDH & $\begin{array}{l}\text { 5'-CACCCACTCCTCCACCTTTG-3' and 5'-CCACCACCCTGTTGC } \\
\text { TGTAG-3' }\end{array}$ \\
\hline
\end{tabular}




\section{RNA interference and overexpression of TRIM14}

TRIM14 short hairpin RNAs (shTRIM14) or control shRNA (shNC) was constructed in PLKO.1 vector (Addgene, Cambridge, MA, USA). The cDNA encoding full-length human TRIM14 was constructed in PLVX-puro Vector (Clontech, Palo Alto, CA, USA). The constructs were confirmed by sequencing. Lentiviruses were produced in 293T cells by contransfecting lentiviral constructs with packaging plasmids for 48-72 h. The collected viral supernatant was filtered through $0.45-\mu \mathrm{m}$ filter, and infected target cell lines in the presence of polybrene $(8 \mu \mathrm{g} / \mathrm{ml}$, Sigma, St. Louis, MO, USA). The shRNA sequence against TRIM14 are shown as follows: shTRIM14\#1: CAGATTACTACTTGA CGAA; shTRIM14\#2: CATTGGACATTCGCCTTAA; shTRIM14\#3: GATCGCTATTGCTGAAATA.

\section{Transwell migration and invasion assays}

Transwell migration assays were performed using Transwell plates with $8 \mu \mathrm{m}$ pore filters (Costar, Manassas, VA, USA). Viral transduced cells were serum-starved overnight. Cells suspended in $0.2 \mathrm{ml}$ serum-free medium were plated into the upper chamber of Transwell plates $\left(5 \times 10^{4}\right.$ per well), and $0.6 \mathrm{ml}$ medium with $10 \% \mathrm{FBS}$ were loaded to the lower chamber. After incubation at $37{ }^{\circ} \mathrm{C}$ for $24 \mathrm{~h}$, cells in the upper chamber were completely removed with a cotton swab, while cells that migrated to the lower chamber were fixed in $4 \%$ paraformaldehyde and stained with $0.1 \%$ crystal violet. Then stained cells were counted in five random fields at $200 \times$ magnification, and the average number was taken. The average number of control group was set as $100 \%$, and relative migration and invasion of other groups was calculated by comparison of the cell number with the average number of control group. For invasion assays, the experiment procedure was similar to migration assays except that the Transwell insert was coated with Matrigel (BD Biosciences, Franklin Lakes, NJ, USA) before the cells were loaded.

\section{Western blot analysis}

RIPA buffer (Beyotime, Shanghai, China) plus protease inhibitor cocktail (Roche, Indianapolis, IN, USA) was used to lysate cultured cells. Equal amount of protein (30 $\mu \mathrm{g}$ per sample) was separated by SDS-polyacrylamide gel electrophoresis (SDS-PAGE) gel and transferred onto the nitrocellulose membrane. The membranes were blocked and probed at $4{ }^{\circ} \mathrm{C}$ overnight with the following primary antibodies: STAT3 (\#9139), and GAPDH (\#5174) from Cell Signaling Technology (Danvers, MA, USA); SPHK1 (Ab71700), p-STAT3 (Ab76315), VEGFA (Ab51745), MMP2 (Ab37150) and MMP9 (Ab38898) from Abcam (Cambridge, MA, USA). The membranes were then probed with horseradish peroxidase (HRP)-conjugated secondary antibody at room temperature for $1 \mathrm{~h}$. Signal was detected by the enhanced chemiluminescence reagents (Thermo Fisher). GAPDH was used as loading control. Representative blots of three independent experiments are shown.

\section{Statistical analysis}

All analyses were done with GraphPad Prism software (La Jolla, CA, USA). Data are presented as mean \pm standard deviation (SD). Significance of statistical analysis was assessed using ANOVA tests. $\mathrm{P}<0.05$ was considered as statistically significant.

\section{Results}

Up-regulation of TRIM14 mRNA expression in CRC tissues

We tested the mRNA expression of TRIM14 in CRC tissues and matched non-cancerous tissues by real-time PCR analysis. TRIM14 mRNA levels were markedly upregulated in CRC tissues (Fig. 1a). Additionally, TRIM14 mRNA was detected in various CRC cell lines including SW620, Caco2, LoVo, HT-29 and SW1116 (Fig. 1b). TRIM14 mRNA was highly expressed in HT-29 and SW620 cell lines, and lowly expressed in LoVo cells.

\section{TRIM14 promoted CRC cell migration and invasion}

We then explored whether TRIM14 expression affected CRC cell migration and invasion. We manipulated TRIM14 expression in CRC cells by lentiviral infection. As indicated in Fig. 2a, b, all the 3 TRIM14 shRNA (shTRIM14\#1, \#2 and \#3) significantly down-regulated TRIM14 expression in both HT-29 and SW620 cells, and shTRIM14\#1 was the most efficiency one and used in the subsequent assays. As Fig. 2c shown, pLVX-TRIM14 markedly up-regulated TRIM14 expression in LoVo cells. Inhibition of TRIM14 in HT-29 (Fig. 3a and Additional file 1: Figure S1A) and SW620 cells (Fig. 3b and Additional file 1: Figure S1B) attenuated cell migration and invasion, whereas ectopic expression of TRIM14 in LoVo cells enhanced invasion and migration (Fig. $3 \mathrm{c}$ and Additional file 1: Figure $\mathrm{S} 1 \mathrm{C}$ ) at $12 \mathrm{~h}$ and $24 \mathrm{~h}$ after plating.

\section{TRIM14 activated the SPHK1/STAT3 signaling pathway in CRC cells}

A recent study has shown that TRIM14 enhanced the STAT3 signaling pathway in breast cancer cells [9], which plays a critical role in CRC progression [14]. SPHK1 is upregulated in CRC cancer patients [16] and leads to constituent activation of STAT3 in colitis-associated CRC [17]. Thus, we examined whether TRIM14 affected the SPHK1/STAT3 signaling in CRC cells. TRIM14 knockdown repressed the levels of SPHK1 and phosphorylated STAT3, while they had no effect on the levels of STAT3 (Fig. 4a). TRIM14 knockdown repressed the mRNA and 

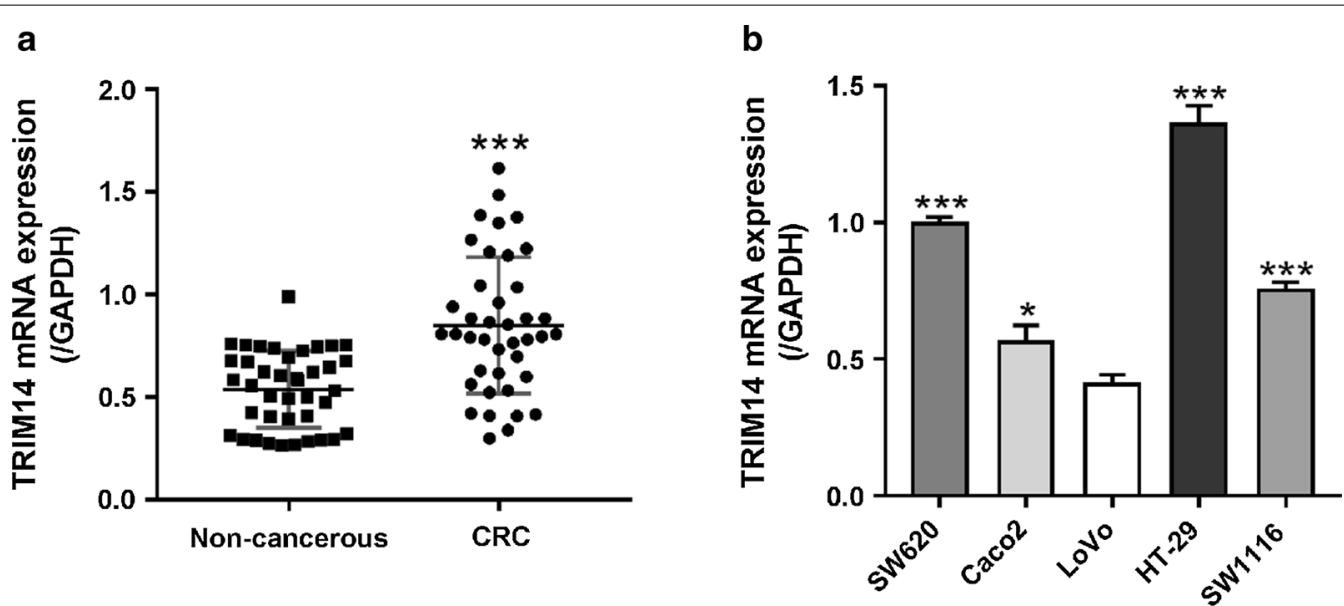

Fig. 1 mRNA expression of TRIM14 in human CRC tissues and CRC cell lines. a Real-time PCR analysis of TRIM14 expression in 40 pairs of non-cancerous colon and CRC tissues. TRIM14 expression were normalized to the expression of GAPDH. ${ }^{* *} \mathrm{P}<0.001$. $\mathbf{b}$ TRIM14 expression in 5 CRC cell lines by real-time PCR analysis. ${ }^{*} \mathrm{P}<0.05,{ }^{* *} \mathrm{P}<0.001$ versus LoVo cells

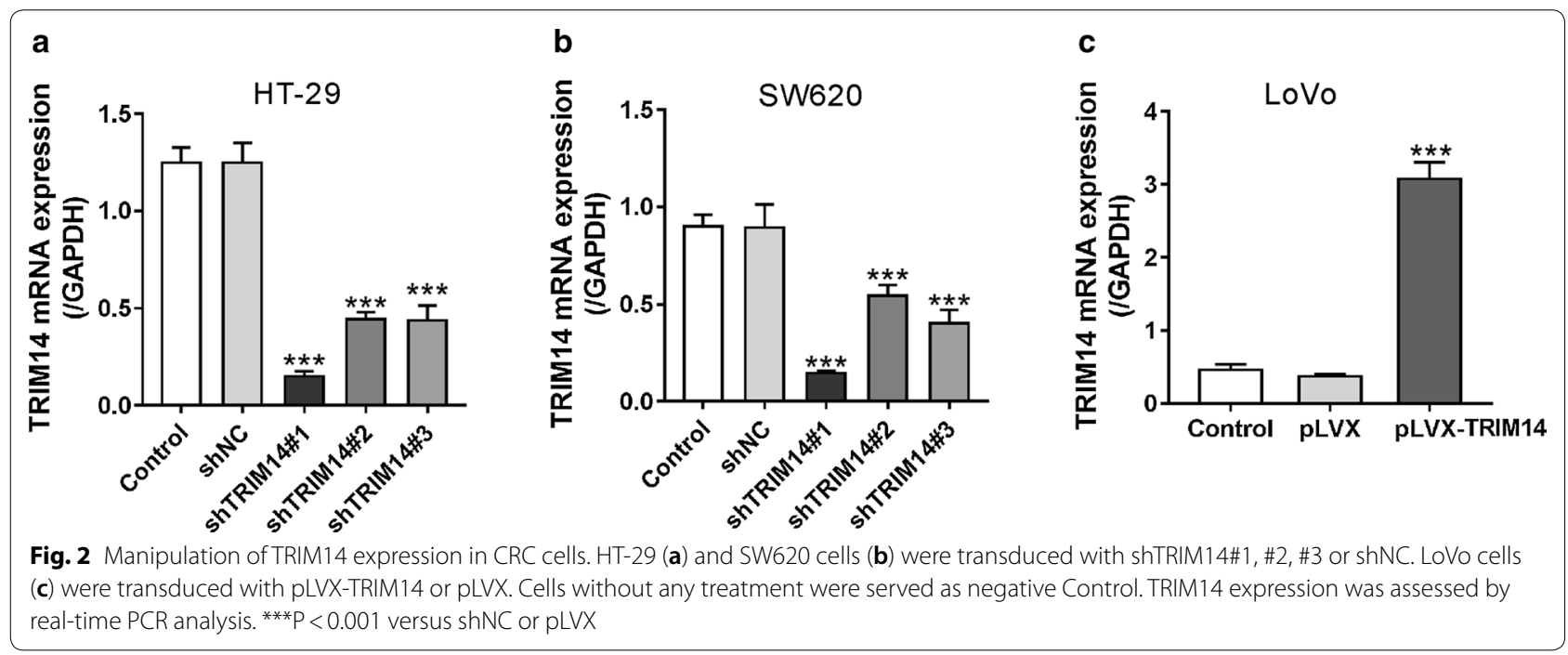

protein levels of MMP2, MMP9 and VEGF (Fig. 4a, b), which are transcriptional targets of the STAT3 signaling pathway [22]. Reverse results were observed in LoVo cells overexpressing TRIM14 (Fig. 4c, d). Collectively, these data suggest that TRIM14 can enhance the SPHK1/ STAT3 signaling cascade in CRC cells.
Inhibition of SPHK1/STAT3 signaling with SKI-II/AG490 counteracted the promontory effects of TRIM14 on CRC cell migration and invasion

To assess whether the effects of TRIM14 on CRC cells were mediated by the SPHK1/STAT3 signaling, LoVo cells were infected with pLVX-TRIM14 virus and treated

(See figure on next page.)

Fig. 3 TRIM14 affected the migration and invasion of CRC cells. HT-29 (a) and SW620 cells (b) were transduced with shTRIM14\#1 or shNC. LoVo cells (c) were transduced with PLVX-TRIM14 or pLVX. Transwell migration and invasion assays were performed to determine the effect of TRIM14 expression on the migration and invasion of CRC cell lines at $24 \mathrm{~h}$ after treatment. Cells without any treatment were served as negative Control. The stained cells were counted in five random fields at $\times 200$ magnification, and the average number was taken. The average number of Control group was set as 100\%, and relative migration and invasion of other groups was calculated by comparison of the cell number with the average number of control group. ${ }^{* *} \mathrm{P}<0.001$ versus shNC or pLVX 

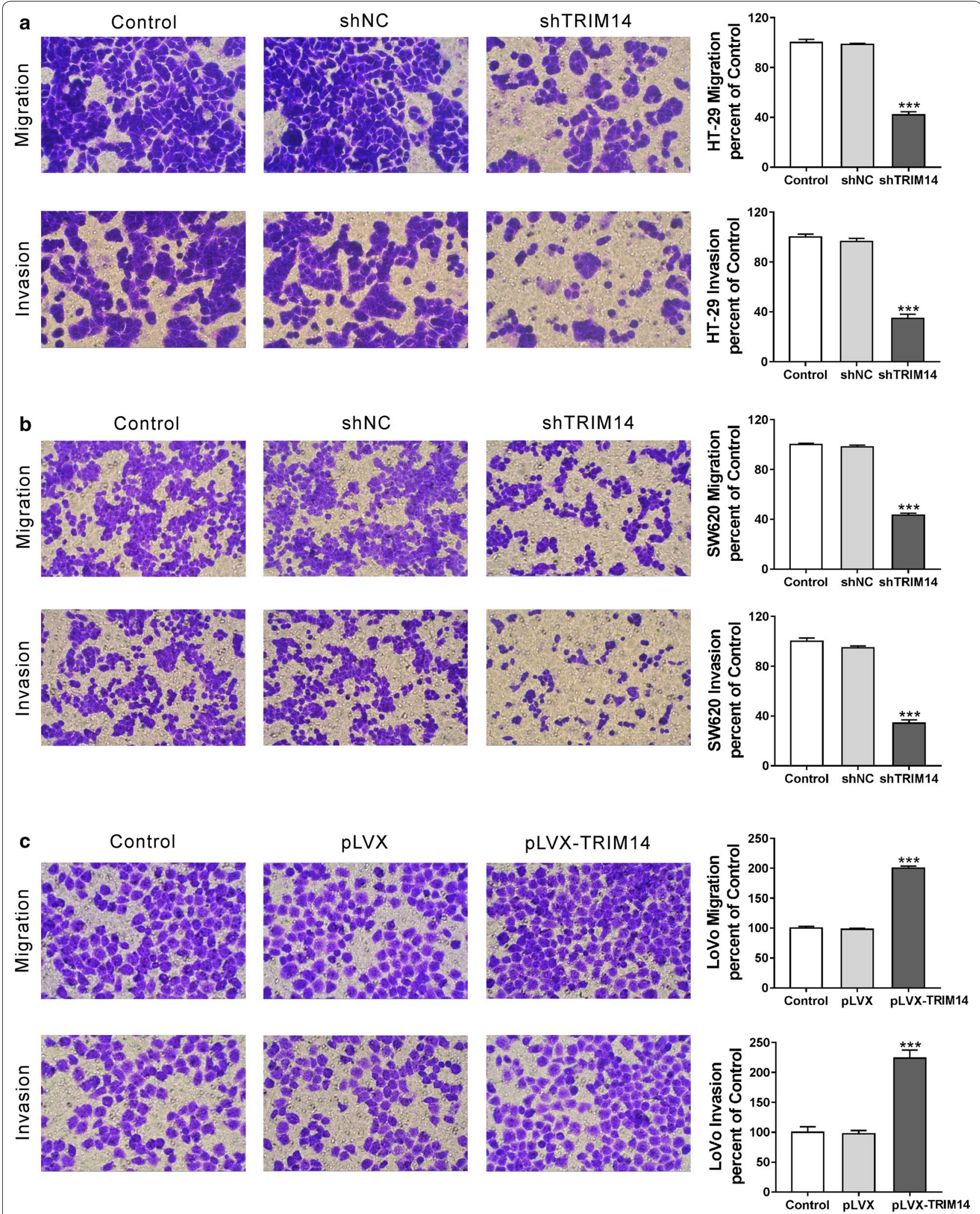

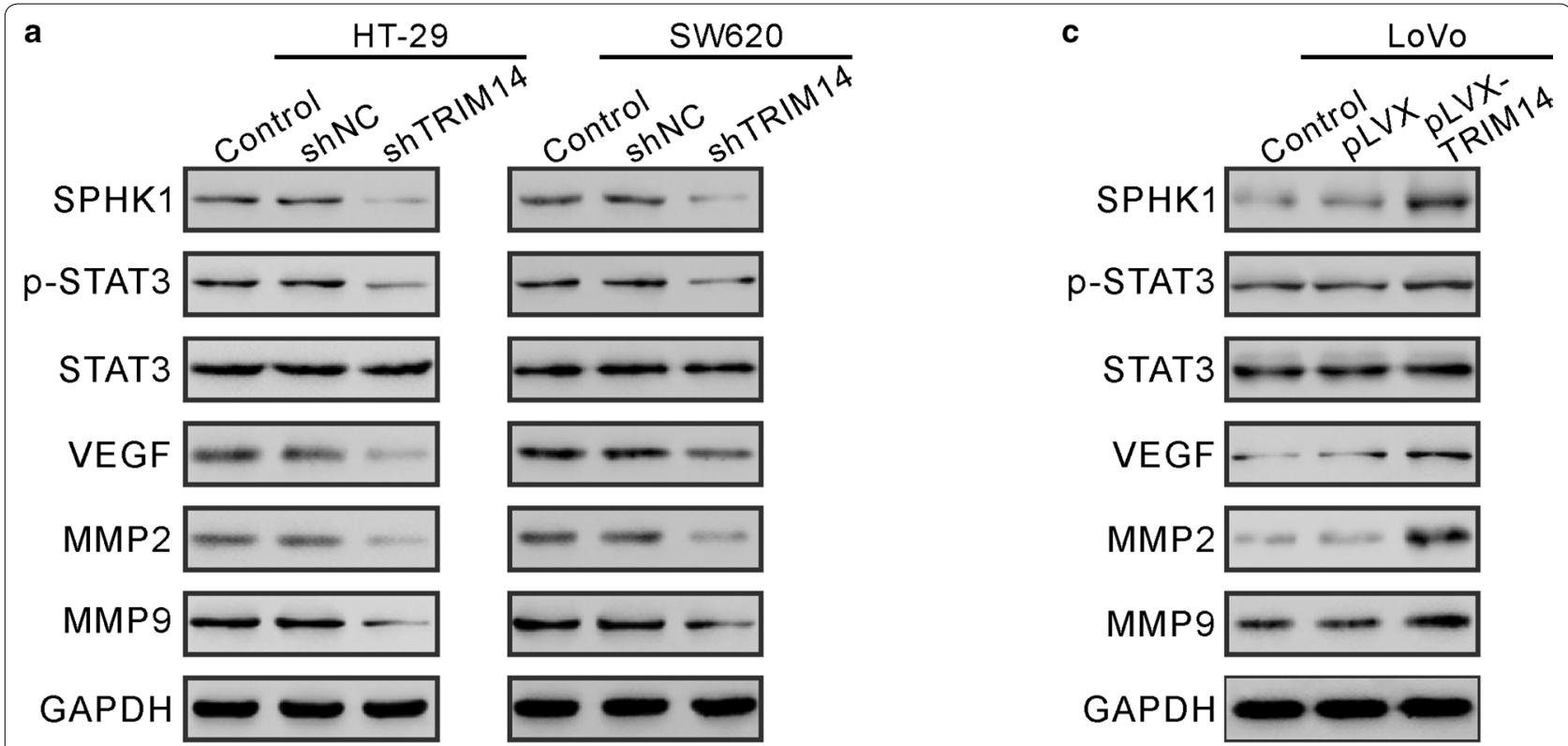

b
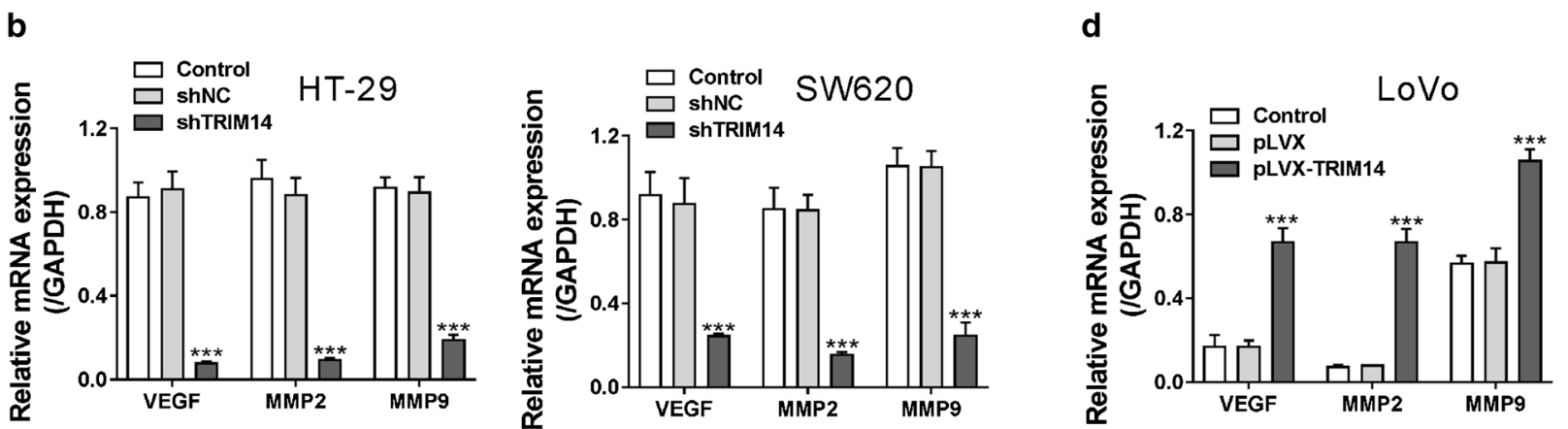

Fig. 4 TRIM14 activated the SPHK1/STAT3 signaling pathway in CRC cells. HT-29 and SW620 cells (a, b) were transduced with shTRIM14\#1 or shNC. LoVo cells $(\mathbf{c}, \mathbf{d})$ were transduced with pLVX-TRIM14 or pLVX. The expression of relevant proteins was detected by real-time PCR and western blotting analyses at $48 \mathrm{~h}$ post transduction. ${ }^{* * *} \mathrm{P}<0.001$ versus shNC or pLVX

with a SPHK1 inhibitor SKI-II [23] or a STAT3 inhibitor AG490. As expected, the inhibition of SPHK1 or STAT3 signaling mostly blocked the promontory effects of TRIM14 on migration and invasion (Fig. 5a), as well as the levels of p-STAT3, VEGF, MMP2 and MMP9 (Fig. 5b).

\section{Discussion}

Evidences have shown that TRIM family proteins participate in the pathogenesis of various cancers [3]. For example, TRIM24 [24, 25] and TRIM29 [26, 27] are upregulated in CRC tissues and exert oncogenic functions on CRC. In the current study, TRIM14 expression was higher in CRC tissues than in paired normal colorectal tissues (Fig. 1). In line with our findings, previous studies have reported the overexpression of TRIM14 in HCC,
OSCC, TSCC, osteosarcoma, glioma and breast cancer [5-10]. On the contrary, decreased TRIM14 expression was observed in NSCLC [4]. These results suggest the different expression pattern and function of TRIM14 in diverse cancers.

Distant metastases contributes to the high mortality rate in CRC patients [1]. Tumor metastasis is a multistage process. Tumor cell migration and invasion are responsible for the infiltration of tumor cells into lymphatic vessels, blood vessels and distant organs, and represent for the key steps during tumor metastasis $[28,29]$. The function of TRIM14 in cancer cell migration and invasion has been described in osteosarcoma [6] and OSCC [7]. Consistently with the above reports, our data showed that TRIM14 knockdown inhibited CRC cell migration and invasion, while TRIM14 overexpression had opposite 


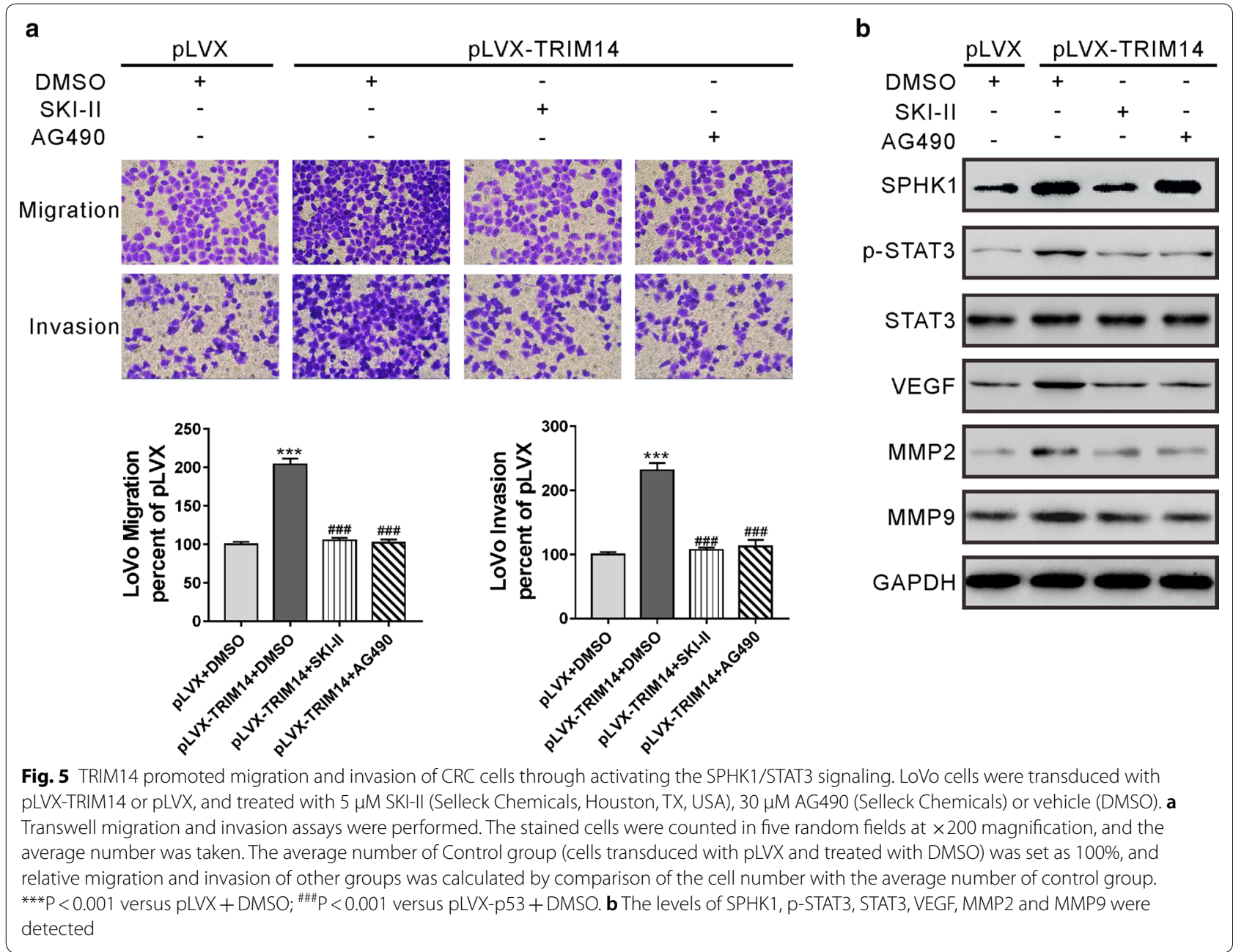

effects (Fig. 3). Our data further demonstrated the important role of TRIM14 in tumorigenesis.

Several cancer-related signaling pathways have been reported to be activated by TRIM14, such as AKT pathway in osteosarcoma [6], NF-kB pathway in TSCC [8], Wnt/ $\beta$-catenin pathway in glioma [10] and STAT3 in breast cancer [9]. The present study focused on the STAT3 pathway, and the effects of TRIM14 on the other pathways will be considered in our future study. STAT3 signaling pathway plays an important role in tumorrelated activities, such as cell proliferation, migration, invasion and metastasis $[11,13]$. Evidence has shown that STAT3 is constitutively activated in CRC [30], which is critical for CRC cell growth, survival, invasion and migration [14]. It has been demonstrated that SPHK1, which catalyzes the formation of sphingosine 1-phosphate (S1P) [15], causes the persistent activation of STAT3 in colitisassociated CRC [17]. In the present study, TRIM14 overexpression promoted CRC cell migration and invasion, which was counteracted by SKI-II or AG490, suggesting that SPHK1/STAT3 contributed to TRIM14-mediated CRC cell migration and invasion. Matrix metalloproteinases (MMPs) are key enzymes responsible for the degradation of extracellular matrix, thus involving in tumor metastasis [31]. Numerous studies have suggested the increased expression and activity of MMP2 and MMP9 in CRC specimens [32-35]. VEGF, an important signal protein, is associated with the progression, invasion and metastasis of CRC and may be independent prognostic marker for CRC patients [36]. VEGF, MMP2 and MMP9 are known as transcriptional targets of the STAT3 signaling pathway [22]. Here, TRIM14 overexpression increased the phosphorylation of STAT3, and the expression of its downstream targets MMP2, MMP9 and VEGF in CRC cells, further demonstrating the involvement of the SPHK1/STAT3 pathway in the function of TRIM14. TRIM14 contains the PRYSPRY domain, which is crucial for protein-protein interactions [37]. A previous study has identified more than 70 TRIM14 interacting proteins, which are associated with protein ubiquitination [4]. We 
speculated that TRIM14 may regulate SPHK1 expression via its interaction partner, although the precise mechanism how TRIM14 regulated the SPHK1/STAT3 pathway requires further investigation.

\section{Conclusions}

This study reveals the increased expression of TRIM14 in CRC samples, and an important role of TRIM14 in CRC cell migration and invasion through the SPHK1/STAT3 signaling. TRIM14 may be a potential molecular target to prevent CRC metastasis.

\section{Additional file}

Additional file 1: Figure S1. TRIM14 affected the migration and invasion of CRC cells. HT-29 (A) and SW620 cells (B) were transduced with shTRIM14\#1 or shNC. LoVo cells (C) were transduced with pLVX-TRIM14 or pLVX. Transwell migration and invasion assays were performed to determine the effect of TRIM14 expression on the migration and invasion of $C R C$ cell lines at $12 \mathrm{~h}$ after treatment. Cells without any treatment were served as negative Control. The stained cells were counted in five random fields at $200 \times$ magnification, and the average number was taken. The average number of Control group was set as 100\%, and relative migration and invasion of other groups was calculated by comparison of the cell number with the average number of control group. ${ }^{* *} \mathrm{P}<0.001$ versus shNC or pLVX.

\section{Abbreviations}

CRC: colorectal cancer; TRIM: tripartite motif; RBCC: RING B-box-coiled-coil; NSCLC: non-small cell lung cancer; HCC: hepatocellular carcinoma; OSCC: oral squamous cell carcinoma; TSCC: tongue squamous cell carcinoma; STAT : signal transducer and activator of transcription; SPHK1: sphingosine kinase 1; S1P: sphingosine 1-phosphate; MMP: matrix metalloproteinase; VEGF: vascular endothelial growth factor; shTRIM14:TRIM14 short hairpin RNAs; shNC: control shRNA; HRP: horseradish peroxidase.

\section{Authors' contributions}

ZHJ and SBW designed the study; ZHJ, HQL, XFH, GRY, XFL and LLZ performed the experiments and prepared the figures; $Z \mathrm{HJ}, \mathrm{HQL}$ and SBW contributed to drafting the manuscript. All authors read and approved the final manuscript.

\section{Acknowledgements}

Not applicable.

\section{Competing interests}

The authors declare that they have no competing interests.

\section{Availability of data and materials}

The datasets used and/or analyzed during the current study are available from the corresponding author on reasonable request.

\section{Consent for publication}

Not applicable.

\section{Ethics approval and consent to participate}

The study was approved by the Ethics Committee of Yiwu Hospital, Wenzhou Medical University (Yiwu, China).

\section{Funding}

This study was supported by grant from Zhejiang Science and Technology Project (2017C33179).

\section{Publisher's Note}

Springer Nature remains neutral with regard to jurisdictional claims in published maps and institutional affiliations.

Received: 16 July 2018 Accepted: 6 December 2018

Published online: 11 December 2018

\section{References}

1. Torre LA, Bray F, Siegel RL, Ferlay J, Lortet-Tieulent J, Jemal A. Global cancer statistics, 2012. CA Cancer J Clin. 2015;65(2):87-108.

2. $\mathrm{Cao} H, \mathrm{XuE}$, Liu H, Wan L, Lai M. Epithelial-mesenchymal transition in colorectal cancer metastasis: a system review. Pathol Res Pract. 2015;211(8):557-69

3. Hatakeyama S. TRIM proteins and cancer. Nat Rev Cancer. 2011;1 1(11):792.

4. Hai J, Zhu CQ, Wang T, Organ SL, Shepherd FA, Tsao MS. TRIM14 is a putative tumor suppressor and regulator of innate immune response in non-small cell lung cancer. Sci Rep. 2017;7:39692.

5. Dong B, Zhang W. High levels of TRIM14 are associated with poor prognosis in hepatocellular carcinoma. Oncol Res Treat. 2018:41(3):129-34.

6. Xu G, Guo Y, Xu D, Wang Y, Shen Y, Wang F, et al. TRIM14 regulates cell proliferation and invasion in osteosarcoma via promotion of the AKT signaling pathway. Sci Rep. 2017;7:42411.

7. Wang T, Ren Y, Liu R, Ma J, Shi Y, Zhang L, et al. miR-195-5p suppresses the proliferation, migration, and invasion of oral squamous cell carcinoma by targeting TRIM14. Biomed Res Int. 2017;2017:7378148.

8. Su X, Wang J, Chen W, Li Z, Fu X, Yang A. Overexpression of TRIM14 promotes tongue squamous cell carcinoma aggressiveness by activating the NF-kappaB signaling pathway. Oncotarget. 2016;7(9):9939-50.

9. Hu G, Pen W, Wang M. TRIM14 promotes breast cancer cell proliferation by inhibiting apoptosis. Oncol Res. 2018;1:1. https://doi. org/10.3727/096504018X15214994641786.

10. Tan Z, Song L, Wu W, Zhou Y, Zhu J, Wu G, et al. TRIM14 promotes chemoresistance in gliomas by activating $\mathrm{Wnt} / \beta$-catenin signaling via stabilizing Dvl2. Oncogene. 2018;37(40):5403-15.

11. Siveen KS, Sikka S, Surana R, Dai X, Zhang J, Kumar AP, et al. Targeting the STAT3 signaling pathway in cancer: role of synthetic and natural inhibitors. Biochim Biophys Acta. 2014;1845(2):136-54.

12. Yu H, Jove R. The STATs of cancer-new molecular targets come of age. Nat Rev Cancer. 2004;4(2):97.

13. Banerjee K, Resat $\mathrm{H}$. Constitutive activation of STAT3 in breast cancer cells: a review. Int J Cancer. 2016:138(11):2570-8.

14. Xiong H, Zhang Z-G, Tian X-Q, Sun D-F, Liang Q-C, Zhang Y-J, et al. Inhibition of JAK1, 2/STAT3 signaling induces apoptosis, cell cycle arrest, and reduces tumor cell invasion in colorectal cancer cells. Neoplasia. 2008;10(3):287-97.

15. Pyne NJ, Pyne S. Sphingosine 1-phosphate and cancer. Nat Rev Cancer. 2010;10(7):489-503

16. Kawamori T, Kaneshiro T, Okumura M, Maalouf S, Uflacker A, Bielawski $J$, et al. Role for sphingosine kinase 1 in colon carcinogenesis. FASEB J. 2009:23(2):405-14.

17. Liang J, Nagahashi M, Kim EY, Harikumar KB, Yamada A, Huang W, et al. Sphingosine-1-phosphate links persistent STAT3 activation, chronic intestinal inflammation, and development of colitis-associated cancer. Cancer Cell. 2013;23(1):107-20.

18. Okumura F, Matsunaga Y, Katayama Y, Nakayama KI, Hatakeyama S. TRIM8 modulates STAT3 activity through negative regulation of PIAS3. I Cell Sci. 2010;123(13):2238-45.

19. Okumura F, Okumura AJ, Matsumoto M, Nakayama KI, Hatakeyama S. TRIM8 regulates Nanog via Hsp90ß-mediated nuclear translocation of STAT3 in embryonic stem cells. Biochim Biophys Acta. 2011:1813(10):1784-92.

20. Zhang C, Mukherjee S, Tucker-Burden C, Ross JL, Chau MJ, Kong J, et al. TRIM8 regulates stemness in glioblastoma through PIAS3-STAT3. Mol Oncol. 2017;11(3):280-94.

21. Livak KJ, Schmittgen TD. Analysis of relative gene expression data using real-time quantitative $P C R$ and the $2^{-\triangle \Delta C T}$ method. Methods. 2001;25(4):402-8 
22. Carpenter RL, Lo H-W. STAT3 target genes relevant to human cancers. Cancers. 2014;6(2):897-925.

23. Tonelli F, Alossaimi M, Williamson L, Tate R, Watson DG, Chan EYW, et al. The sphingosine kinase inhibitor 2-(p-hyroxyanilino)-4-(p-chlorophenyl) thiazole reduces androgen receptor expression via an oxidative stressdependent mechanism. Br J Pharmacol. 2013;168(6):1497-505.

24. Wang J, Zhu J, Dong M, Yu H, Dai X, Li K. Knockdown of tripartite motif containing 24 by lentivirus suppresses cell growth and induces apoptosis in human colorectal cancer cells. Oncol Res. 2014:22(1):39-45.

25. Wang F-Q, Han Y, Yao W, Yu J. Prognostic relevance of tripartite motif containing 24 expression in colorectal cancer. Pathol Res Pract. 2017;213(10):1271-5.

26. Jiang $T$, Tang HM, Lu S, Yan DW, Yang YX, Peng ZH. Up-regulation of tripartite motif-containing 29 promotes cancer cell proliferation and predicts poor survival in colorectal cancer. Med Oncol. 2013;30(4):715.

27. Xu W, Xu B, Yao Y, Yu X, Cao H, Zhang J, et al. RNA interference against TRIM29 inhibits migration and invasion of colorectal cancer cells. Oncol Rep. 2016;36(3):1411-8.

28. Valastyan S, Weinberg RA. Tumor metastasis: molecular insights and evolving paradigms. Cell. 2011:147(2):275-92.

29. Steeg PS. Tumor metastasis: mechanistic insights and clinical challenges. Nat Med. 2006:12(8):895.

30. Spano J-P, Milano G, Rixe C, Fagard R. JAK/STAT signalling pathway in colorectal cancer: a new biological target with therapeutic implications. Eur J Cancer. 2006:42(16):2668-70.

31. Deryugina El, Quigley JP. Matrix metalloproteinases and tumor metastasis Cancer Metastasis Rev. 2006;25(1):9-34.

32. Roeb E, Dietrich CG, Winograd R, Arndt M, Breuer B, Fass J, et al. Activity and cellular origin of gelatinases in patients with colon and rectal carcinoma. Cancer. 2001;92(10):2680-91.
33. Collins H, Morris T, Watson S. Spectrum of matrix metalloproteinase expression in primary and metastatic colon cancer: relationship to the tissue inhibitors of metalloproteinases and membrane type-1-matrix metalloproteinase. Br J Cancer. 2001;84(12):1664.

34. Liabakk N-B, Talbot I, Smith RA, Wilkinson K, Balkwill F. Matrix metalloprotease 2 (MMP-2) and matrix metalloprotease 9 (MMP-9) type IV collagenases in colorectal cancer. Cancer Res. 1996:56(1):190-6.

35. Karakiulakis G, Papanikolaou C, Jankovic S, Aletras A, Papakonstantinou

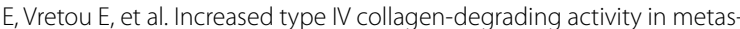
tases originating from primary tumors of the human colon. Invasion Metastasis. 1997;17(3):158-68.

36. Ishigami S, Arii S, Furutani M, Niwano M, Harada T, Mizumoto M, et al. Predictive value of vascular endothelial growth factor (VEGF) in metastasis and prognosis of human colorectal cancer. Br J Cancer. 1998;78(10):1379.

37. Zhou Z, Jia X, Xue Q, Dou Z, Ma Y, Zhao Z, et al. TRIM14 is a mitochondrial adaptor that facilitates retinoic acid-inducible gene-l-like receptor-mediated innate immune response. Proc Natl Acad Sci USA. 2014;111(2):E245-54
Ready to submit your research? Choose BMC and benefit from:

- fast, convenient online submission

- thorough peer review by experienced researchers in your field

- rapid publication on acceptance

- support for research data, including large and complex data types

- gold Open Access which fosters wider collaboration and increased citations

- maximum visibility for your research: over $100 \mathrm{M}$ website views per year

At BMC, research is always in progress.

Learn more biomedcentral.com/submissions 